\title{
Propofol pretreatment attenuates lipopolysaccharide-induced acute lung injury in rats by activating the phosphoinositide-3-kinase/Akt pathway
}

\author{
L.L. Zhao ${ }^{1}$, G.C. Hü ${ }^{2}$, S.S. Zhu ${ }^{1}$, J.F. Li ${ }^{3}$ and G.J. $\mathrm{Liu}^{1}$ \\ ${ }^{1}$ Department of Anesthesiology, The Affiliated Hospital of Xuzhou Medical College, Xuzhou, Jiangsu Province, China \\ ${ }^{2}$ Department of Pharmacology, College of Medicine, University of Illinois at Chicago, Chicago, IL, USA \\ ${ }^{3}$ Department of Anesthesiology, Tengzhou Central People's Hospital, Liaocheng, Shandong Province, China
}

\begin{abstract}
The aim of this study was to investigate the effect of propofol pretreatment on lipopolysaccharide (LPS)-induced acute lung injury (ALI) and the role of the phosphoinositide-3-kinase/protein kinase B (PI3K/Akt) pathway in this procedure. Survival was determined $48 \mathrm{~h}$ after LPS injection. At $1 \mathrm{~h}$ after LPS challenge, the lung wet- to dry-weight ratio was examined, and concentrations of protein, tumor necrosis factor- $\alpha$ (TNF- $\alpha$ ), and interleukin- 6 (IL-6) in bronchoalveolar lavage fluid (BALF) were determined using the bicinchoninic acid method or ELISA. Lung injury was assayed via lung histological examination. PI3K and p-Akt expression levels in the lung tissue were determined by Western blotting. Propofol pretreatment prolonged survival, decreased the concentrations of protein, TNF- $\alpha$, and IL- 6 in BALF, attenuated ALI, and increased PI3K and p-Akt expression in the lung tissue of LPS-challenged rats, whereas treatment with wortmannin, a PI3K/Akt pathway specific inhibitor, blunted this effect. Our study indicates that propofol pretreatment attenuated LPS-induced ALI, partly by activation of the PI3K/Akt pathway.
\end{abstract}

Key words: Acute lung injury; Propofol; Lipopolysaccharide; PI3K/Akt pathway

\section{Introduction}

Acute lung injury $(A L I)$ and acute respiratory distress syndrome (ARDS) often occur in severe diseases such as serious infection, shock, trauma, and burn, and are clinically characterized by progressive hypoxemia and respiratory distress syndrome, contributing to diffuse pulmonary interstitial and alveolar edema caused by pulmonary capillary endothelial cells and alveolar epithelial cell injury. ALI and ARDS, as the major causes of acute respiratory failure, increase the risk of morbidity and mortality in severely affected patients $(1,2)$. Despite intensive investigation in animals and patients, the etiology and mechanisms of ALI/ ARDS have not been clarified. At present, studies suggest that the systemic inflammatory response caused by many kinds of pathogenic factors plays a critical role (3). However, the defined cellular and molecular mechanisms underlying ALI/ARDS have not been fully explored.

It is known that the phosphoinositide-3-kinase/protein kinase $\mathrm{B}$ (PI3K/Akt) pathway exists extensively in cells, and is involved in the regulation of a series of physiological activities such as cell apoptosis, proliferation and differentiation, and metabolism (4). Recent studies have shown that activation of the PI3K/Akt pathway can prevent lung epithelial cell death induced by oxidants, inhibit lung epithelial cell apoptosis, and significantly delay the occurrence of $A L I$ and improve animal survival rate $(5,6)$. Our work, as well as that of others, has identified that pharmacological inhibition of phosphatase and tensin homolog (PTEN), a major suppressor of the PI3K/Akt pathway, can enhance wound closure and restore lung epithelial monolayer integrity following injury (7).

Propofol (2,6-di-isopropylphenol) is a potent intravenous anesthetic and sedative agent widely used to facilitate intubation and ventilation (8). Recently, many studies have shown that propofol has a protective effect on endotoxin-induced ALI via mechanisms such as suppression of the migration, phagocytosis, and oxidative

Correspondence: G.J. Liu, Department of Anesthesiology, The Affiliated Hospital of Xuzhou Medical College, No. 99 Huaihai Western Road, Xuzhou 221002, Jiangsu Province, China. Fax: +86-516-8560-9999. E-mail: liugongjian61@hotmail.com 
ability of macrophages, inhibition of the release of inflammatory mediators, and alleviation of damage of alveolar epithelial and endothelial cells $(9,10)$. In addition, increasingly, research has shown that propofol plays an important role in the protective effects against ischemic injury by activating the PI3K/Akt pathway (11). However, few studies have investigated the effect of propofol pretreatment on the PI3K/Akt pathway, and whether it might attenuate lipopolysaccharide (LPS)-induced ALI.

The aim of this study was to investigate whether propofol pretreatment attenuates LPS-induced ALI in rats and to test the hypothesis that these beneficial actions are mediated by activation of the PI3K/Akt pathway.

\section{Material and Methods}

All experimental protocols were carried out with the approval of the Animal Care and Use Committee of Xuzhou Medical College (Xuzhou, Jiangsu Province, China) and in accordance with the National Institutes of Health (USA) Guide for Care and Use of Laboratory Animals (Publication No. 80-23, revised 1996).

\section{Animal preparation}

Adult male Sprague-Dawley rats (weighing 250 to $300 \mathrm{~g}$ ) were provided by the Experimental Animal Center of Xuzhou Medical College, [license number: SYXK (Jiangsu) 2002-0038]. Animals were housed in a temperature $\left(23 \pm 1^{\circ} \mathrm{C}\right)$ controlled room, under a $12: 12-\mathrm{h}$ light-dark cycle (lights on from 8:00 am to 8:00 pm), and with food and water ad libitum. Prior to the experiments, animals were allowed to habituate to the housing facilities for at least 1 week, and all efforts were made to minimize animal suffering. Thirty-six rats were randomly divided into 6 groups as follows: control group (saline only), propofol group (20 $\mathrm{mg} \cdot \mathrm{kg}^{-1} \cdot \mathrm{h}^{-1}$ propofol for $2 \mathrm{~h}$, iv; Fresenius Kabi, China) (12), wortmannin group (0.6 mg/kg wortmannin, iv; Sigma Aldrich, USA), LPS group (5 mg/kg Escherichia coli B55:5, iv; Sigma Aldrich), propofol + LPS group $\left(20 \mathrm{mg} \cdot \mathrm{kg}^{-1} \cdot \mathrm{h}^{-1}\right.$ propofol was administered $1 \mathrm{~h}$ before LPS, and followed by infusion of $20 \mathrm{mg} \cdot \mathrm{kg}^{-1} \cdot \mathrm{h}^{-1}$ propofol for $1 \mathrm{~h}$ ), and wortmannin + propofol + LPS group $(0.6 \mathrm{mg} / \mathrm{kg}$ wortmannin was administered $30 \mathrm{~min}$ before infusion of propofol, and followed by the same protocol as the propofol + LPS group). The dose and administration of the drugs were based on previous studies (7) and on our preliminary studies.

To determine the mortality of LPS-challenged rats with propofol and wortmannin treatment (iv), we employed another 36 rats and injected them with $5 \mathrm{mg} / \mathrm{kg}$ LPS (iv) and treated them with $20 \mathrm{mg} \cdot \mathrm{kg}^{-1} \cdot \mathrm{h}^{-1}$ propofol (administered $1 \mathrm{~h}$ before LPS, followed by $20 \mathrm{mg} \cdot \mathrm{kg}^{-1} \cdot \mathrm{h}^{-1}$ propofol for $1 \mathrm{~h}, \mathrm{n}=12)$ or wortmannin $(0.6 \mathrm{mg} / \mathrm{kg}$ administered $30 \mathrm{~min}$ before propofol and followed by the same protocol as the propofol + LPS group, $n=12$ ). Survival was determined $48 \mathrm{~h}$ after LPS injection.
The rats were euthanized with a lethal injection of pentobarbital $1 \mathrm{~h}$ after the LPS challenge, the thorax was opened, and the lungs were immediately removed en bloc. The superior lobe of the right lung was harvested for lung water content measurement [by determining the ratio of the wet to dry (W/D) weight of the lung tissue], and the right lung was weighed, dried in an oven at $60^{\circ} \mathrm{C}$ for $72 \mathrm{~h}$, and reweighed.

\section{Lung lavage analysis}

The left lung harvested from each rat was infused with $5 \mathrm{~mL}$ ice-cold PBS, which was withdrawn and reinfused two more times. There were no differences in the volume of bronchoalveolar lavage fluid (BALF) recovered $(4.2 \pm 0.4 \mathrm{~mL}$ fluid) after the lung lavage process among the 6 groups, via centrifugation at $1200 \mathrm{~g}$ for $10 \mathrm{~min}$ at $4^{\circ} \mathrm{C}$. The supernatant was collected and stored at $-80^{\circ} \mathrm{C}$ for batch analysis. The concentration of proteins in BALF was analyzed via the bicinchoninic acid (BCA) method, and the levels of tumor necrosis factor (TNF)- $\alpha$ and interleukin (IL)- 6 were determined using rat TNF- $\alpha$ and IL6 ELISA kits (both from Boster Biological Engineering Co. Ltd., China), according to the manufacturer's instructions. Samples taken from each animal (6 per group) were analyzed in triplicate for TNF- $\alpha$ and IL-6.

\section{Lung histological examination}

The middle lobe of the right lung from each animal was immersed in $10 \%$ neutral phosphate-buffered formalin fixative for $24 \mathrm{~h}$ and embedded in paraffin. A series of $4-\mu \mathrm{m}$ sections were cut and stained with hematoxylin and eosin. Images were captured with a Nikon SMZ 1500 Microscope (Nikon, Japan) to observe histological changes. Lung sections were analyzed using standard histological techniques. Moreover, ALI was scored by a blinded observer according to the following four items (13): alveolar congestion, hemorrhage, infiltration or aggregation of neutrophils in the airspace or vessel wall, and thickness of the alveolar wall.

\section{Western blotting}

PI3K and p-Akt in the lung tissue were detected by Western blot analysis. Protein was extracted from the inferior lobe of the right lung, and the protein content was determined by the BCA method. Cytosolic fractions were separated by SDS-PAGE, transferred, and immobilized on a nitrocellulose membrane (BioRad, USA). The membrane was blocked by incubation with $5 \%$ nonfat dried milk in PBS for $2 \mathrm{~h}$ at room temperature and then incubated with anti-PI3K (1:500, Cell Signaling, USA) or anti-p-Akt (1:500, Cell Signaling) overnight at $4^{\circ} \mathrm{C}$. After they were washed 3 times in phosphate buffer saline with Tween-20 (PBST), the membranes were incubated with 1:2000 alkaline phosphatase-conjugated anti-rabbit antibody (v/v; Santa Cruz Biotechnology, USA) for $2 \mathrm{~h}$ at room temperature. The immune complexes were detected 


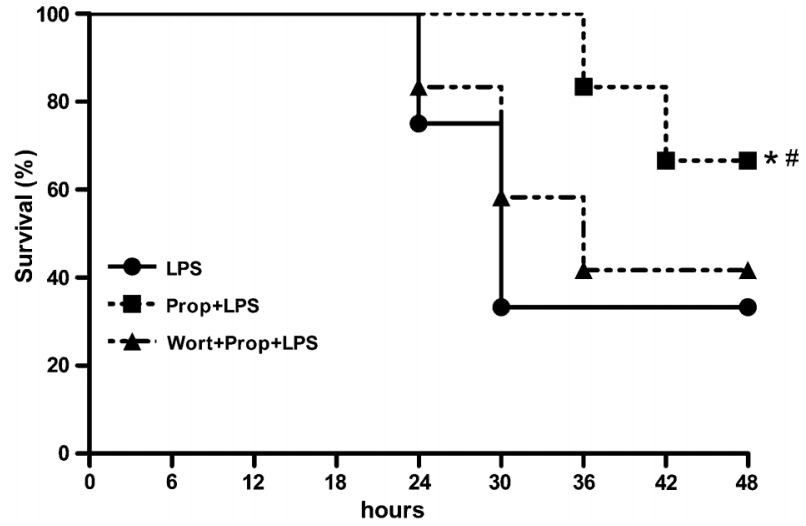

Figure 1. Survival curve of LPS-challenged rats combined with saline or propofol pretreatment. Data are reported as means \pm SD for $n=12$ animals per experimental group. LPS: lipopolysaccharide; Prop: propofol; Wort: wortmannin. ${ }^{*} \mathrm{P}<0.05$ compared to the LPS group; ${ }^{\#} \mathrm{P}<0.05$ compared to the Wort + Prop + LPS group (log-rank test).

using a nitro blue tetrazolium/5-bromo-4-chloro-3-indolyl phosphate assay kit (Sigma). Western blot densitometry analysis of signal intensity was performed using the Adobe Photoshop software (Adobe, USA). The levels of PI3K and p-Akt from densitometry were normalized to $\beta$-actin levels.

\section{Statistical analysis}

The SPSS 13.0 software (SPSS Inc., USA) was used for all statistical analyses. Data are reported as means $\pm S D$, and $n$ is the number of animals in each study group. Multiple parametric comparisons were performed by one-way analysis of variance, followed by the Tukey post hoc test when differences were significant. Comparisons among mortality rates of the groups were made with the Kaplan-Meier and Mantel-Cox methods. Values of $\mathrm{P}<0.05$ were considered to be statistically significant.

\section{Results}

\section{Effect of propofol pretreatment on mortality rate of LPS-treated rats}

Three hours after the LPS challenge, rats manifested lethargy, piloerection, diarrhea, huddling, and malaise, clear signs of endotoxic shock, as in previous studies (14). At $48 \mathrm{~h}$ after LPS injection, the mortality rates were $66.67 \%, 58.33 \%$, and $33.33 \%$ for the LPS, propofol, and wortmannin treatment groups, respectively. The propofol pretreatment group had a significantly lower mortality rate than the other two groups $(P<0.05, n=12$ animals per group); however, there was no significant difference between the LPS and wortmannin treatment groups (Figure 1). This observation suggests that propofol at least partially decreases the mortality rate caused by LPS injection, which can be partially attenuated by wortmannin treatment.

\section{Effect of propofol pretreatment on lung W/D ratio, and concentration of proteins, TNF- $\alpha$, and IL- 6 in BALF of LPS-treated rats}

One hour after the LPS challenge, the lung W/D ratio and concentration of proteins, TNF- $\alpha$, and IL- 6 in BALF were analyzed in order to detect the lung injury of LPS-treated
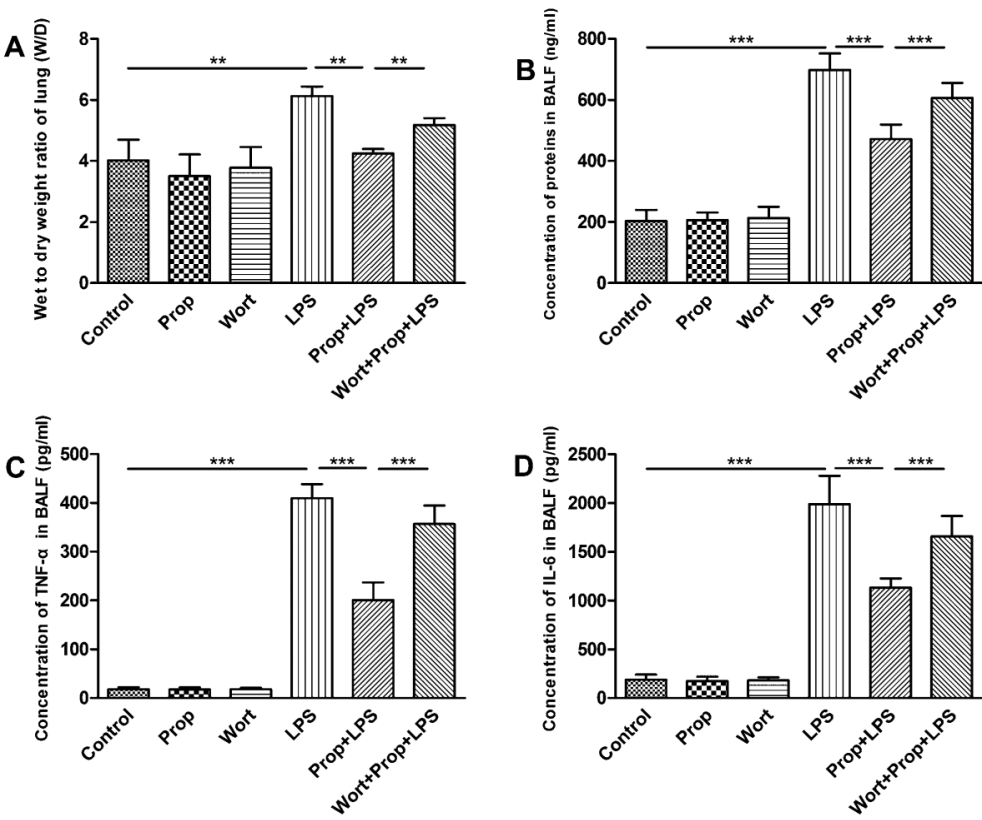

Figure 2. The lung wet to dry weight (W/D) ratio, and concentration of proteins, TNF- $\alpha$ and IL-6 in bronchoalveolar lavage fluid (BALF). Data are reported as means $\pm S D$ for $n=8$ animals per group. Data are representative of triplicate experiments with similar results. TNF$\alpha$ : tumor necrosis factor- $\alpha$; IL-6: interleukin-6; LPS: lipopolysaccharide; Prop: propofol; Wort: wortmannin. ${ }^{* *} \mathrm{P}<0.01,{ }^{* *} \mathrm{P}<0.001$ (Tukey multiple comparison test) 

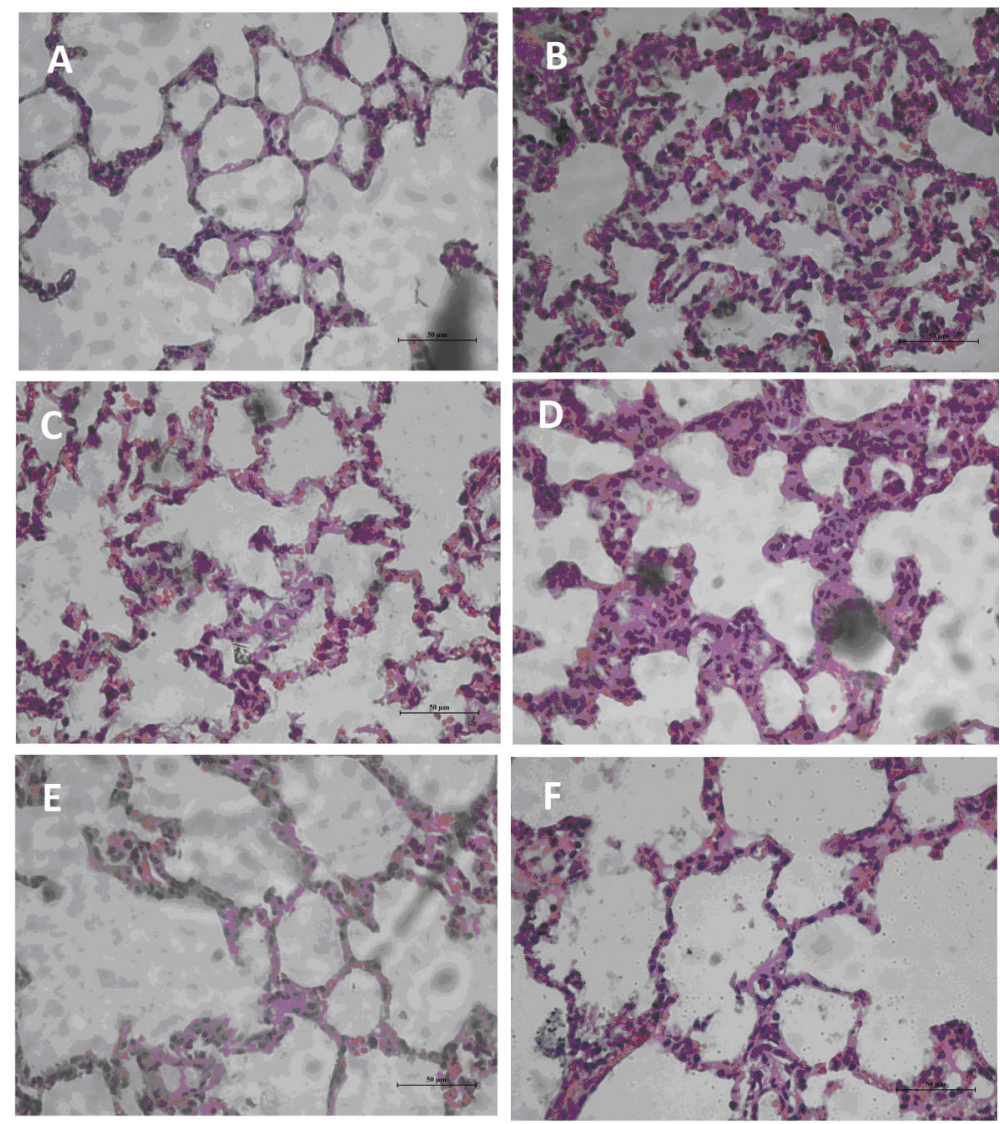

Figure 3. Histopathology of representative lung sections. Sections were stained with hematoxylin and eosin. $A$, Control group. $B$, Lipopolysaccharide (LPS) group: edematous changes of alveolar walls, swelling of alveolar epithelial cells, and massive polymorphonuclear infiltration were observed. C, Propofol + LPS group: less damage was observed compared to the LPS group. $D$, Wortmannin + propofol + LPS group: edematous changes of alveolar walls, swelling of alveolar epithelial cells, and massive polymorphonuclear infiltration were observed. $E$, Propofol group: no differences were observed compared to the control group. $F$, Wortmannin group: no differences were observed compared to the control group. Magnification bar: $50 \mu \mathrm{m}$ $(400 \times)$. rats. As shown in Figure 2, lung W/D ratio and levels of proteins, TNF- $\alpha$, and IL- 6 in BALF was significantly higher in the LPS group than in the control group $(\mathrm{P}<0.001, \mathrm{n}=6$ animals per group); however, there were no significant differences of W/D ratio, concentration of proteins, TNF- $\alpha$, and IL-6 in BALF among the control, propofol, and wortmannin groups. Propofol pretreatment significantly decreased the W/D ratio and the concentration of proteins, TNF- $\alpha$, and IL- 6 in BALF caused by LPS injection, while treatment with wortmannin partially blunted the effect of propofol pretreatment (values of $P$ from 0.05 to $0.01, n=6$ animals per group).

\section{Effect of propofol pretreatment on lung injury of LPS-treated rats}

As shown in Figure 3, there were no obvious inflammatory changes to the lung tissues in the control, propofol, and wortmannin groups. Histopathology showed more alveolar edema, hemorrhage, wall thickening and hyperinflation, and infiltration of significant numbers of inflammatory cells into the alveolar spaces and interstitial spaces in the LPS group. These features of ALI were significantly attenuated in the propofol + LPS group, manifested as less alveolar septal thickening, less inflammatory cell infiltration, and less alveolar congestion. However, alveolar septal thickening, inflammatory cell infiltration, and alveolar congestion were exacerbated by wortmannin treatment.

\section{Effect of propofol pretreatment on protein expression} of PI3K and p-Akt in lung tissues of LPS-treated rats

We also examined protein expression of PI3K and p-Akt in rat lung tissues by Western blot analysis of lung homogenates. PI3K and p-Akt protein expression was detected in all lung tissue samples from control and experimental rats, as shown in Figure 4. Compared to the control group, $\mathrm{PI} 3 \mathrm{~K}$ and $\mathrm{p}$-Akt protein expression levels were significantly decreased in the LPS and wortmannin groups; however, the levels of PI3K and p-Akt protein expression were elevated in the propofol group $(P<0.05$ or $\mathrm{P}<0.01, \mathrm{n}=6$ animals per group). Propofol pretreatment significantly reversed the effect of LPS injection on expression of PI3K and p-Akt protein in the propofol + LPS group, which was alleviated by wortmannin treatment in the wortmannin + propofol + LPS group $(\mathrm{P}<0.05$ or $\mathrm{P}<0.01$, $n=6$ animals per group). Our data suggested that propofol reversed the effect of the LPS injection-induced decrease in the levels of PI3K and p-Akt protein expression, while wortmannin treatment blunted this effect. 


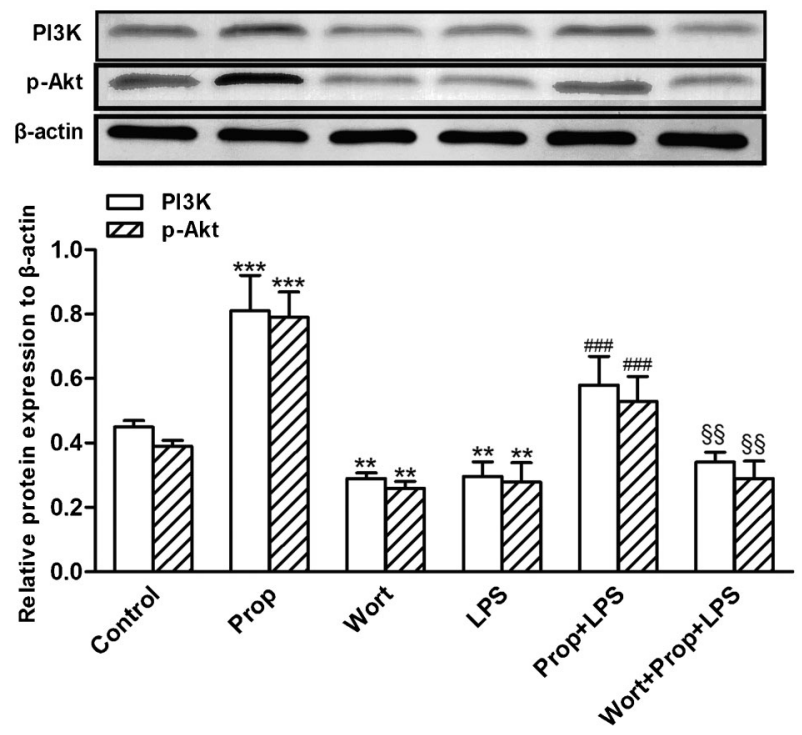

Figure 4. Phosphoinositide-3-kinase $(\mathrm{PI} 3 \mathrm{~K})$ and $\mathrm{p}$-Akt protein in rat lung. Top, Representative Western blots are shown with the $\mathrm{PI} / \mathrm{K}, \mathrm{p}$-Akt and $\beta$-actin labeled band. Bottom, Densitometry of all Western blot results from rat lungs of the 6 groups. Data are reported as means $\pm S D$ for $n=6$ animals per group. LPS: lipopolysaccharide; Prop: propofol; Wort: wortmannin. ${ }^{* *} \mathrm{P}<0.01$, ${ }^{* * *} \mathrm{P}<0.001$ compared to the control group; $\# \# \# \mathrm{P}<0.001$ compared to the LPS group; ${ }^{\S \S} \mathrm{P}<0.01$ compared to the Prop + LPS group (Tukey multiple comparison test).

\section{Discussion}

ALI and its more severe form, ARDS, characterized by a cascade amplifying inflammation and a secondary diffuse lung parenchyma injury caused by a variety of inflammatory mediators and effector cells, are relatively common syndromes in critically ill patients, associated with high morbidity and mortality (15). A major cause of the development of ALI is sepsis, and LPS is implicated as an important toxin that precipitates lung injury. Propofol, a widely used intravenous anesthetic and sedative agent used to sedate intubated, mechanically ventilated ALI and

\section{References}

1. Brun-Buisson C, Minelli C, Bertolini G, Brazzi L, Pimentel J, Lewandowski K, et al. Epidemiology and outcome of acute lung injury in European intensive care units. Results from the ALIVE study. Intensive Care Med 2004; 30: 51-61, doi: 10.1007/s00134-003-2022-6.

2. Stapleton RD, Wang BM, Hudson LD, Rubenfeld GD, Caldwell ES, Steinberg KP. Causes and timing of death in patients with ARDS. Chest 2005; 128: 525-532, doi: 10.1378/chest.128.2.525.

3. Herold S, Gabrielli NM, Vadasz I. Novel concepts of acute lung injury and alveolar-capillary barrier dysfunction. Am J Physiol Lung Cell Mol Physiol 2013; 305: L665-L681.
ARDS patients, contributes to the rapid onset and short duration of action, and rapid elimination. A number of studies have found that propofol attenuates endotoxininduced ALI in a murine model of sepsis (16-18) and inhibits LPS-induced nitric oxide production and biosynthesis of the inflammatory cytokines, TNF- $\alpha$, interleukin (IL)-1 $\beta$, and IL-6 in macrophages (19). The anti-inflammatory effects of propofol have attracted growing attention; however, the molecular mechanisms for these effects are still unclear.

It has been reported that the PI3K/Akt pathway exists extensively in cells and is involved in the regulation of a series of physiological activities such as cell apoptosis, proliferation and differentiation, and metabolism (4). Consistent with studies indicating that activation of the PI3K/Akt pathway significantly delays the occurrence of ALI and improves animal survival rate $(5,6)$, our research and that of others has identified that pharmacological inhibition of PTEN, a major suppressor of the PI3K/Akt pathway, can enhance wound closure and restore lung epithelial monolayer integrity following injury (7). We speculated that propofol pretreatment could protect against LPS-induced ALI via activation of the PI3K/Akt pathway. In the present study, we found that levels of p-Akt protein expression in lung tissue significantly decreased, with a large amount of protein leakage and the release of inflammatory cytokines, and an increase in water content in the lung in LPS-induced ALI. Propofol pretreatment could reverse the effect of a LPS challenge, suggesting that activation of the PI3K/Akt pathway may repair damage to alveolar epithelium and the capillary endothelial barrier, which was supported by our further research that wortmannin, a specific inhibitor of the PI3K/ Akt pathway, could partially attenuate the protective effects of propofol pretreatment on LPS-induced ALI.

In summary, LPS-induced downregulation of the PI3K/ Akt pathway exacerbated ALI. Propofol improved ALI and upregulation of the PI3K/Akt pathway. Therefore, activation of the PI3K/Akt pathway may be the molecular mechanism that allows propofol to protect against ALI in endotoxic shock.

4. Garat CV, Crossno JT Jr, Sullivan TM, Reusch JE, Klemm DJ. Inhibition of phosphatidylinositol 3-kinase/Akt signaling attenuates hypoxia-induced pulmonary artery remodeling and suppresses CREB depletion in arterial smooth muscle cells. J Cardiovasc Pharmacol 2013; 62: 539-548, doi 10.1097/FJC.0000000000000014.

5. Ray P, Devaux Y, Stolz DB, Yarlagadda M, Watkins SC, Lu $Y$, et al. Inducible expression of keratinocyte growth factor (KGF) in mice inhibits lung epithelial cell death induced by hyperoxia. Proc Natl Acad Sci U S A 2003; 100: 6098-6103, doi: 10.1073/pnas.1031851100.

6. Zhang X, Shan P, Alam J, Fu XY, Lee PJ. Carbon monoxide 
differentially modulates STAT1 and STAT3 and inhibits apoptosis via a phosphatidylinositol 3-kinase/Akt and p38 kinase-dependent STAT3 pathway during anoxia-reoxygenation injury. J Biol Chem 2005; 280: 8714-8721, doi: 10.1074/jbc.M408092200.

7. Lai JP, Dalton JT, Knoell DL. Phosphatase and tensin homologue deleted on chromosome ten (PTEN) as a molecular target in lung epithelial wound repair. $\mathrm{Br} J$ Pharmacol 2007; 152: 1172-1184.

8. Marik PE. Propofol: therapeutic indications and side-effects. Curr Pharm Des 2004; 10: 3639-3649, doi: 10.2174/ 1381612043382846.

9. Tanaka T, Takabuchi S, Nishi K, Oda S, Wakamatsu T, Daijo $\mathrm{H}$, et al. The intravenous anesthetic propofol inhibits lipopolysaccharide-induced hypoxia-inducible factor 1 activation and suppresses the glucose metabolism in macrophages. $J$ Anesth 2010; 24: 54-60, doi: 10.1007/s00540-009-0829-1.

10. Wang L, Wu B, Sun Y, Xu T, Zhang X, Zhou M, et al. Translocation of protein kinase $C$ isoforms is involved in propofol-induced endothelial nitric oxide synthase activation. Br J Anaesth 2010; 104: 606-612, doi: 10.1093/bja/ aeq064.

11. Wang HY, Wang GL, Yu YH, Wang Y. The role of phosphoinositide-3-kinase/Akt pathway in propofol-induced postconditioning against focal cerebral ischemia-reperfusion injury in rats. Brain Res 2009; 1297: 177-184.

12. Liu S, Feng G, Wang GL, Liu GJ. p38MAPK inhibition attenuates LPS-induced acute lung injury involvement of NF-kappaB pathway. Eur J Pharmacol 2008; 584: 159-165, doi: 10.1016/j.ejphar.2008.02.009.
13. Ma L, Wu XY, Zhang LH, Chen WM, Uchiyama A, Mashimo $\mathrm{T}$, et al. Propofol exerts anti-inflammatory effects in rats with lipopolysaccharide-induced acute lung injury by inhibition of CD14 and TLR4 expression. Braz J Med Biol Res 2013; 46: 299-305, doi: 10.1590/1414-431X20122379.

14. Yeh $\mathrm{CH}$, Cho W, So EC, Chu CC, Lin MC, Wang JJ, et al. Propofol inhibits lipopolysaccharide-induced lung epithelial cell injury by reducing hypoxia-inducible factor-1alpha expression. $\mathrm{Br} J$ Anaesth 2011; 106: 590-599, doi: 10. 1093/bja/aer005.

15. Rubenfeld GD, Caldwell E, Peabody E, Weaver J, Martin $\mathrm{DP}$, Neff $\mathrm{M}$, et al. Incidence and outcomes of acute lung injury. N Engl J Med 2005; 353: 1685-1693, doi: 10.1056/ NEJMoa050333.

16. Chu CH, David LD, Hsu YH, Lee KC, Chen HI. Propofol exerts protective effects on the acute lung injury induced by endotoxin in rats. Pulm Pharmacol Ther 2007; 20: 503-512, doi: 10.1016/j.pupt.2006.03.006.

17. Takao $\mathrm{Y}$, Mikawa K, Nishina K, Obara H. Attenuation of acute lung injury with propofol in endotoxemia. Anesth Analg 2005; 100: 810-6, , table, doi: 10.1213/01.ANE. 0000144775.19385.8C.

18. Gao J, Zeng BX, Zhou LJ, Yuan SY. Protective effects of early treatment with propofol on endotoxin-induced acute lung injury in rats. $\mathrm{Br} J$ Anaesth 2004; 92: 277-279, doi: 10.1093/bja/aeh050.

19. Li HH, Lin YC, Chen PJ, Hsiao CH, Lee JY, Chen WC, et al. Interleukin-19 upregulates keratinocyte growth factor and is associated with psoriasis. $\mathrm{Br} J$ Dermatol 2005; 153: 591595, doi: 10.1111/j.1365-2133.2005.06665.x. 